\title{
ON A THEOREM OF PLESSNER
}

\author{
BY W. C. RANDELS $\dagger$
}

Plessner $\ddagger$ has shown that if $f(x) \subset L_{2}$ on $(-\pi, \pi)$ and

$$
f(x) \sim \frac{a_{0}}{2}+\sum_{n=1}^{\infty}\left(a_{n} \cos n x+b_{n} \sin n x\right),
$$

then

$$
\sum_{n=2}^{\infty}\left(a_{n} \cos n x+b_{n} \sin n x\right)(\log n)^{-1 / 2}
$$

converges almost everywhere on $(-\pi, \pi)$. We designate the set where (1) converges by $E(P l, f)$. This set is then known to be of measure $2 \pi$. The sets $E(F, f)$, consisting of the points where

$$
\phi(t)=f(x+t)+f(x-t)-2 f(x) \rightarrow 0, \text { as } t \rightarrow 0,
$$

and $E(L, f)$, consisting of the points where

$$
\Phi(t)=\int_{0}^{t}|\phi(\tau)| d \tau=o(t), \text { as } t \rightarrow 0,
$$

are of much importance in the theory of Fourier series. The set $E(L, f)$ is known to be of measure $2 \pi$ for all integrable functions. It is obvious that

$$
E(F, t) \subset E(L, f) .
$$

We propose in this note to investigate the inclusion relationships between these sets and $E(P l, f)$. We shall prove

$$
E(F, f) \nsubseteq E(P l, f),
$$

and

$$
E(P l, f) ф E(L, f) .
$$

We first consider (2). Plessner $\S$ showed that, if (1) converges,

† Sterling Research Fellow.

$\ddagger$ A. Plessner, Journal für Mathematik, vol. 155 (1926), pp. 15-25.

\&oc. cit., p. 22. 
(4) $S_{n}(x)=\frac{a_{0}}{2}+\sum_{\nu=1}^{n}\left(a_{\nu} \cos \nu x+b_{\nu} \sin \nu x\right)=o\left\{(\log n)^{1 / 2}\right\}$.

However, it is well known $\dagger$ that, for a continuous function, the estimate

$$
S_{n}(x)=o(\log n)
$$

cannot be improved. This implies that (4) need not be satisfied at every point of continuity and hence

$$
E(F, f) \nsubseteq E(P l, f) .
$$

In order to prove (3) we shall construct a function $f(x) \subset L_{2}$ on $(-\pi, \pi)$ for which (1) converges at $x=0$ but such that

$$
\int_{0}^{t}|\phi(\tau)| d \tau \neq o(t) \text { as } t \rightarrow 0 \text {. }
$$

The function is similar to one constructed by Paley $\ddagger$ for another purpose. We define $f(x)$ by

$$
f(x)=\left\{\begin{array}{l}
x\left\{\left(x-n^{-1}\right) n \log n\right\}^{-1}, \text { if } n^{-2} \geqq\left|x-n^{-1}\right| \geqq n^{-3}, \\
0, \text { elsewhere on }(0, \pi), \\
f(-x) \text { for } 0 \geqq x \geqq-\pi .4, \cdots),
\end{array}\right.
$$

Then, since

$$
\begin{aligned}
\int_{0}^{\pi}|f(x)|^{2} d x & =O\left\{\sum_{n=3}^{\infty} n^{-4}(\log n)^{-2} \int_{n^{-3}}^{n^{-2}} \frac{d x}{x^{2}}\right\} \\
& =O\left\{\sum_{n=1}^{\infty} n^{-1}(\log n)^{-2}\right\},
\end{aligned}
$$

$f(x) \subset L_{2}$ on $(-\pi, \pi)$. We have at $x=0, \phi(t)=2 f(t)$ and

$$
\int_{0}^{t}|\phi(t)| d t>\sum_{n=[1 / t]+1}^{\infty}\left(n^{2} \log n\right)^{-1} \int_{n^{-2}}^{n^{-3}} \frac{d x}{x}=\sum_{n=[1 / t]+1}^{\infty} n^{-2}>\frac{t}{3}
$$

$\dagger$ P. Du Bois Reymond, Abhandlungen der Bayerische Akademie, vol. 12, part 2 (1876). There is a simplification of Du Bois Reymond's method given by Lebesgue, Leçons sur les Séries Trigonométriques, 1906, pp. 84-86.

$\ddagger$ R. E. A. C. Paley, Proceedings Cambridge Philosophical Society, vol. 26 (1930), pp. 173-203; see \$10, pp. 201-203. 
for $t$ sufficiently small. Now we consider $S_{m}(0)$. It is well known that

$$
\begin{aligned}
S_{m}(0) & =\frac{1}{2 \pi} \int_{0}^{\pi} \phi(t) \frac{\sin (m+1 / 2) t}{\sin t / 2} d t \\
& =\frac{1}{\pi} \int_{0}^{\pi} \phi(t) \frac{\sin m t}{t} d t+O(1),
\end{aligned}
$$

while

$$
\begin{aligned}
S_{m}^{*}(0) \equiv & \frac{1}{\pi} \int_{0}^{\pi} \phi(t) \frac{\sin m t}{t} d t \\
= & \frac{1}{\pi} \sum_{n=3}^{\infty} \frac{2}{n \log n}\left\{\int_{n^{-1}-n^{-2}}^{n^{-1}-n^{-3}} \frac{\sin m t}{\left(t-n^{-1}\right)} d t\right. \\
& \left.+\int_{n^{-1}+n^{-3}}^{n^{-1}+n^{-2}} \frac{\sin m t}{\left(t-n^{-1}\right)} d t\right\}
\end{aligned}
$$

But

$$
\begin{aligned}
\sin m t= & \sin \left(m n^{-1}\right) \cos \left(m\left(t-n^{-1}\right)\right) \\
& +\sin \left(m\left(t-n^{-1}\right)\right) \cos \left(m n^{-1}\right),
\end{aligned}
$$

so that

$$
\begin{aligned}
\pi S_{m}{ }^{*}(0) & =4 \sum_{n=3}^{\infty}(n \log n)^{-1} \cos \left(m n^{-1}\right) \int_{n^{-3}}^{n^{-2}} \frac{\sin m t}{t} d t \\
& =4\left\{\sum_{n=3}^{\left[m^{1 / 3}\right]}+\sum_{n=\left[m^{1 / 3}\right]+1}^{\left[m^{1 / 2}\right]}+\sum_{n=\left[m^{1 / 2}\right]+1}^{\infty}\right\} \\
& \equiv I_{1}+I_{2}+I_{3} .
\end{aligned}
$$

Now, if $m a<1, a>b>0$,

$$
\int_{b}^{a} \frac{\sin m t}{t} d t=\int_{m b}^{m a} \frac{\sin m t}{t} d t=O\{m a\},
$$

and, if $m b>1, a>b>0$,

$$
\int_{b}^{a} \frac{\sin m t}{t} d t=\int_{m b}^{m a} \frac{\sin t}{t} d t=O\left\{\frac{1}{m b}\right\}=O(1) .
$$


Hence

$$
\begin{aligned}
I_{1} & =O\left\{\sum_{n=3}^{\left[m^{1 / 3}\right]}(n \log n)^{-1} \frac{n^{3}}{m}\right\}=O\left\{\frac{1}{m} \sum_{n=3}^{\left[m^{1 / 3}\right]} \frac{n^{2}}{\log n}\right\}=o(1), \\
I_{2} & =O\left\{\sum_{n=\left[m^{1 / 3}\right]+1}^{\left[m^{1 / 2}\right]}(n \log n)^{-1}\right\} \\
& =O\left\{\log \log m^{1 / 2}-\log \log m^{1 / 3}\right\}=O(1) \\
I_{3} & =O\left\{m \sum_{n=\left[m^{1 / 2}\right]+1}^{\infty}(n \log n)^{-1} n^{-2}\right\} \\
& =O\left\{m \sum_{n=\left[m^{1 / 2}\right]+1}^{\infty} n^{-3}\right\}=o(1) .
\end{aligned}
$$

Therefore

$$
S_{m}(0)=S_{m}^{*}(0)+O(1)=O(1) .
$$

We now apply Abel's partial summation to (1) and get

$$
\begin{aligned}
& \sum_{n=2}^{\infty}\left(S_{n}-S_{n-1}\right)(\log n)^{-1 / 2} \\
& =S_{1}(\log 2)^{-1 / 2}+\sum_{n=2}^{\infty} S_{n}\left\{(\log n)^{-1 / 2}-[\log (n+1)]^{-1 / 2}\right\},
\end{aligned}
$$

since $S_{m}(0)=O(1)$ and $(\log n)^{-1 / 2} \rightarrow 0$ as $n \rightarrow \infty$. But since $\log n$ is monotone,

$$
\sum_{n=2}^{\infty}\left\{\mid(\log n)^{-1 / 2}-\left(\log [n+1]^{-1 / 2} \mid\right\}=(\log 2)^{-1 / 2},\right.
$$

and therefore (7) converges. This means that the point $x=0$ is contained in $E(P l, f)$. But since we have already seen that the point $x=0$ is not contained in $E(L, f)$, this proves that

$$
E(P l, f) \nsubseteq E(L, f) .
$$

YALE University 